\title{
Combination chemotherapy of S-1 and low-dose cisplatin for advanced gastric cancer
}

\author{
Shunichi Tsujitani, Kenji Fukuda, and Nobuaki Kaibara \\ Department of Surgery, Division of Surgical Oncology, School of Medicine, Tottori University, 36-1 Nishi-cho, Yonago 683-8504, Japan
}

\begin{abstract}
Background. A previous phase II study showed that S-1 (TS$\left.\mathbf{1}^{\circledR}\right)$ was effective for advanced gastric cancers and had mild toxicity. The present study aimed to evaluate the efficacy and feasibility of this novel anticancer drug combined with lowdose cisplatin (CDDP).

Methods. Fifteen patients with unresectable and recurrent gastric cancer were enrolled. S-1 was administered orally twice daily after meals, at a standard dose of $80 \mathrm{mg} / \mathrm{m}^{2}$ per day according to the late phase II trial protocol. One course consisted of 28 days' consecutive administration followed by 14 days' rest. Five or $10 \mathrm{mg}$ CDDP was infused three times each week (days $1,3,5)$ during $S-1$ administration on hospitalization, and once each week (day 1) at the outpatient clinic. Patients' backgrounds, response rates, response durations, and time to progression were investigated.

Results. None of the 15 patients had a complete response and 8 had a partial response. Therefore, the overall response rate was $53 \%(8 / 15)$. For site efficacy, the response rate was $50 \%$ $(5 / 10)$ for the primary lesion, $50 \%$ (3/6) for liver metastasis, and $39 \%(5 / 13)$ for lymph node metastasis. The median response duration of the 8 responders was 4 months, and the time to progression was 3.3 months. Adverse reactions appeared in $60 \%(9 / 15)$. The incidence of adverse reactions of grades 3 and 4 was 13\% (2/15) and $0 \%$, respectively. As for hematological toxicity, grade 3 leukopenia was observed in 2 patients (2/15), but decreased hemoglobin level and thrombocytopenia did not appear. Although gastrointestinal adverse reactions appeared in $40 \%(6 / 15)$, all reactions were grades 1 and 2. Because of the mild toxicity, most patients were treated at the outpatient clinic.

Conclusions. Combination chemotherapy of S-1 and low-dose CDDP is expected to be a useful chemotherapy for advanced gastric cancer.
\end{abstract}

Key words S-1 • Cisplatin • Combination chemotherapy • Advanced gastric carcinoma

Offprint requests to: $\mathrm{N}$. Kaibara

Received: October 8, 2002 / Accepted: January 28, 2003

\section{Introduction}

Adenocarcinoma of the stomach remains one of the leading causes of cancer death worldwide, and the prognosis of patients with unresectable and recurrent gastric cancer is especially poor. Although complete resection is the only curative therapy for gastric cancer, systemic chemotherapy is necessary for patients with advanced disease. After many chemotherapeutic agents and various administration methods have been reported, it is considered that gastric cancer shows low sensitivity to chemotherapy [1-3]. Several agents, however, have been shown to be relatively effective against gastric cancer, including 5-fluorouracil (5-FU), mitomycin $\mathrm{C}$ (MMC), doxorubicin (ADM), and cisplatin (CDDP), which, individually, achieve a response rate of less than $25 \%$ [4-6]. Some regimens of combination chemotherapy with high response rates have recently been reported for advanced gastric cancer [7-12]. A 5-FU and low-dose CDDP regimen is currently widely used due to its high efficacy and low toxicity [13-15].

$\mathrm{S}-1$ is a novel oral fluorouracil formulation, composed of tegafur (FT), gimestat (CDHP), and otastat potassium (Oxo) in a molar ratio of $1: 0.4: 1$, based on the biochemical modulation of 5-FU [16,17]. CDHP inhibits dihydropyrimidine dehydrogenase (DPD), an enzyme which degrades 5-FU, and maintains prolonged 5-FU concentrations in the blood and tumors [17]. Oxo is distributed in the gastrointestinal tract at a high concentration after oral administration, and alleviates the gastrointestinal toxicity of 5-FU [18]. Early and late phase II clinical trials $[19,20]$ have already been conducted in Japan. The response rates were 54\% (15/28) in the early phase II clinical trial, and 49\% (25/51) in the late one. The incidence of toxicity was $78 \%(40 / 51)$ and that of toxicity of grades 3 and 4 was $20 \%(10 / 51)$ in the late phase II clinical trial [20]. In each trial, the major adverse reactions were myelosuppression and gastrointestinal symptoms. 
Since the introduction of this anticancer drug, a combination of S-1 and low-dose CDDP has been tested in our department as one of the candidates for first-line chemotherapy for unresectable and recurrent gastric cancer. We carried out a retrospective study for evaluating the efficacy and safety of S-1 and CDDP for gastric cancer. Here we report the results of this trial, along with two case reports.

\section{Patients and methods}

\section{Patients}

From July 1999 to June 2002, 18 patients with gastric cancer were treated with a combination of S-1 and low-dose CDDP as a first-line chemotherapy at Tottori University Hospital. The enrollment criteria for this combination chemotherapy were: patients with unresectable or recurrent gastric cancer who consented to the study after getting sufficient information. The subjects of this study were selected from these 18 patients, using eligibility criteria similar to those of the previous phase II study: (1) histologically proven unresectable or recurrent gastric cancer with measurable or assessable lesions; (2) age 20 years or more but less than 80; (3) performance status (PS) of WHO grade 2 or less; (4) life expectancy of 3 months or more; (5) adequate organ function, including bone marrow (white blood cells, $\geq 4000 / \mathrm{mm}^{3}$; platelets, $\geq 100000 / \mathrm{mm}^{3}$ ), liver (serum bilirubin level, $\leq 1.5 \mathrm{mg} / \mathrm{dl}$; serum transaminase level, $\leq 100 \mathrm{U} / \mathrm{l}$ ), and kidney (serum creatinine $\leq$ upper limit of normal range); and (6) no serious complications. Of the 18 patients who were given the drugs, 2 patients had no measurable or assessable lesions and 1 patient had a PS of grade 3. After the application of these criteria, 15 patients remained as the subjects for this study.

The tumor characteristics and staging were defined according to the Japanese classification of gastric carcinoma [21].

\section{Treatment schedule}

The treatment schedule for S- 1 was the same as that used in the previous phase II study; namely, S-1 was administered orally twice daily after meals. Three initial doses of S-1 were established according to the body surface area (BSA), as follows: BSA less than $1.25 \mathrm{~m}^{2}$, $80 \mathrm{mg} /$ day; BSA $1.25 \mathrm{~m}^{2}$ to less than $1.5 \mathrm{~m}^{2}, 100 \mathrm{mg} /$ day; BSA $1.5 \mathrm{~m}^{2}$ or more, $120 \mathrm{mg} /$ day. One course consisted of consecutive administration for 28 days followed by 14 days' rest. Low-dose CDDP was infused for biochemical modulation. Its initial doses were also established according to the BSA, as follows: BSA less than $1.5 \mathrm{~m}^{2}$, $5 \mathrm{mg}$ /day; BSA $1.5 \mathrm{~m}$ or more, $10 \mathrm{mg} /$ day. CDDP was used three times each week (days 1, 3, 5) during S-1 administration on hospitalization, and once each week (day 1) at the outpatient clinic. For some patients who complained of mild fatigue in the outpatient clinic, S-1 was administered for 14 days followed by 7 days' rest as one cycle. In these patients, two cycles were defined as one course, and CDDP was infused once every week (day 1) during S-1 administration. If an unacceptable degree of toxicity became apparent, the chemotherapy was postponed or the dose of S-1 was reduced. Granulocyte colony-stimulating factor (G-CSF) was administered when necessary. When progression of the disease was observed, this combination chemotherapy was stopped and changed to another one.

\section{Evaluation of responses and adverse reactions}

Objective responses to chemotherapy were evaluated according to the World Health Organization standard criteria for measurable metastatic lesions [22]. The responses of the primary lesions were evaluated according to the criteria recommended by the Japanese Research Society for Gastric Cancer [23]. The examinations for the evaluation of the chemotherapy were performed at least once for every two courses of treatment. Patients were followed up every 2 weeks to assess any toxic side effects either on hospitalization or in the outpatient clinic. For the assessment of adverse reactions, the Japan Clinical Oncology Group (JCOG) toxicity grading criteria were adopted [24].

\section{Results}

\section{Patients' characteristics}

The patients' characteristics are summarized in Table 1. The median age was 69 years (range, 37-79 years). Most

Table 1. Patients' characteristics

\begin{tabular}{llc}
\hline Characteristics & & $n=15$ \\
\hline Age (years) & Median & 69 \\
& Range & $37-79$ \\
Sex & Male & 9 \\
& Female & 6 \\
Performance status & 0 & 9 \\
& 1 & 5 \\
Prior gastrectomy & 2 & 1 \\
& Yes & 1 \\
Palliative surgery & No & 14 \\
& Gastrectomy & 4 \\
Metastatic site & Gastro-jejunostomy & 2 \\
& Peritoneum & 5 \\
& Liver & 6 \\
Evaluable sites & Lymph node & 13 \\
& Primary & 10 \\
& Liver & 6 \\
& Lymph node & 13 \\
\hline
\end{tabular}


Table 2. Clinical profiles and results of treatment

\begin{tabular}{rccclcc}
\hline Case no. & Sex & $\begin{array}{c}\text { No. of } \\
\text { courses }\end{array}$ & Response & $\begin{array}{c}\text { Target } \\
\text { lesions }\end{array}$ & $\begin{array}{c}\text { TTP } \\
\text { (months) }\end{array}$ & $\begin{array}{c}\text { Survival } \\
\text { (moths) }\end{array}$ \\
\hline 1 & F & 3 & PR & P,N & 1 & 4 \\
2 & M & 5 & NC & P,N & 5 & $12^{+}$ \\
3 & M & 2 & PD & P,N,D & 1 & 3 \\
4 & M & 3 & NC & P,N,H & 3 & $3^{+}$ \\
5 & F & 3 & PD & P,N,H & 1 & 20 \\
6 & M & 1 & PR & P,N,H,D & 2 & 5 \\
7 & F & 7 & PR & D & 10 & 16 \\
8 & F & 2 & NC & N & 5 & 9 \\
9 & M & 3 & PR & P,N,D & 5 & 9 \\
10 & M & 3 & PR & N,H & 4 & 13 \\
11 & M & 3 & NC & N & 7 & 13 \\
12 & M & 3 & PR & P,N,H & 5 & 13 \\
13 & M & 2 & PD & D & 1 & 5 \\
14 & F & 1 & PR & P,N & 2 & $2^{+}$ \\
15 & F & 2 & PR & P,N,H & 3 & $3^{+}$ \\
\hline
\end{tabular}

TTP, Time to progression; PR, partial response; NC, no change; PD, progressive disease; $\mathrm{P}$, primary lesion; $\mathrm{N}$, nodal metastasis; $\mathrm{H}$, hepatic metastasis; $\mathrm{D}$, dissemination to the peritoneum; + , alive

Table 3. Responses

\begin{tabular}{lccccc}
\hline & CR & PR & NC & PD & RR \\
\hline Overall & 0 & 8 & 4 & 3 & $53.3 \%(8 / 15)$ \\
Primary & 0 & 5 & 4 & 1 & $50.0 \%(5 / 10)$ \\
Metastatic site & & & & & \\
$\quad$ Liver & 0 & 3 & 2 & 1 & $50.0 \%(3 / 6)$ \\
$\quad$ Lymph node & 0 & 5 & 7 & 1 & $38.5 \%(5 / 13)$ \\
\hline
\end{tabular}

$\mathrm{CR}$, Complete response; PR, partial response; NC, no change; PD, progressive disease; $R R$, response rate

of the patients had a good performance status ( 0 or 1$)$ at the initiation of the chemotherapy. One of the 15 patients had a history of gastrectomy. A palliative operation was performed in 6 patients before chemotherapy, and 2 patients were given this combination chemotherapy as a neoadjuvant therapy. Thirteen patients $(87 \%)$ had measurable metastatic lesions. The sites of metastasis were the lymph nodes in 13 patients $(87 \%)$, the liver in $6(40 \%)$, and the peritoneum in $5(33 \%)$. Peritoneal dissemination was diagnosed by the presence of ascites or peritoneal tumors on computed tomography (CT) or ultrasound sonography (USG) examinations, or by confirmation through laparotomy or laparoscopy. None of the patients had received prior chemotherapy before the current treatment.

\section{Response rate}

Detailed clinical profiles and results of treatment are listed in Table 2. The median number of courses of treatment administration was three (range, 1-7). Of the 15 patients in this study, none had a complete response and 8 had a partial response, giving a response rate of
Table 4. Adverse reactions

\begin{tabular}{lllllllcr}
\hline & \multicolumn{3}{c}{ Grade } & & & $\begin{array}{c}\text { Incidence of } \\
\text { grades 3-4 }\end{array}$ \\
\cline { 2 - 3 } \cline { 8 - 9 } Adverse reaction & 1 & 2 & 3 & 4 & & $n$ & Percentage \\
\hline Leukopenia & 2 & 2 & 2 & 0 & & $2 / 15$ & $13.3 \%$ \\
Anemia & 0 & 0 & 0 & 0 & $0 / 15$ & $0 \%$ \\
Nausea/vomiting & 4 & 2 & 0 & 0 & $0 / 15$ & $0 \%$ \\
Diarrhea & 0 & 0 & 0 & 0 & $0 / 15$ & $0 \%$ \\
Renal dysfunction & 0 & 0 & 0 & 0 & $0 / 15$ & $0 \%$ \\
\hline
\end{tabular}

$53 \%(8 / 15)$. For site efficacy, the response rate was $50 \%(5 / 10)$ for the primary lesion, $50 \%(3 / 6)$ for liver metastasis, and 39\% (5/13) for lymph node metastasis (Table 3). The median response duration of the 8 responders was 4 months (range, $1-10$ months). The average time to progression for all patients was 3.3 months.

\section{Adverse reactions}

The major adverse reactions are shown in Table 4. Adverse reactions appeared in $60 \%(9 / 15)$ of the patients. The incidence of adverse reactions of grades 3 and 4 was $13 \%(2 / 15)$ and $0 \%$, respectively. As for hematological toxicity, grade 3 leukopenia was observed in 2 patients (2/15), but decreased hemoglobin level and thrombocytopenia did not appear. Although gastrointestinal adverse reactions appeared in $40 \%(6 / 15)$, all reactions were grades 1 or 2 . During this study, there were no deaths due to toxicity.

As some patients in this study showed remarkable tumor regression, we would like to describe two such cases. 

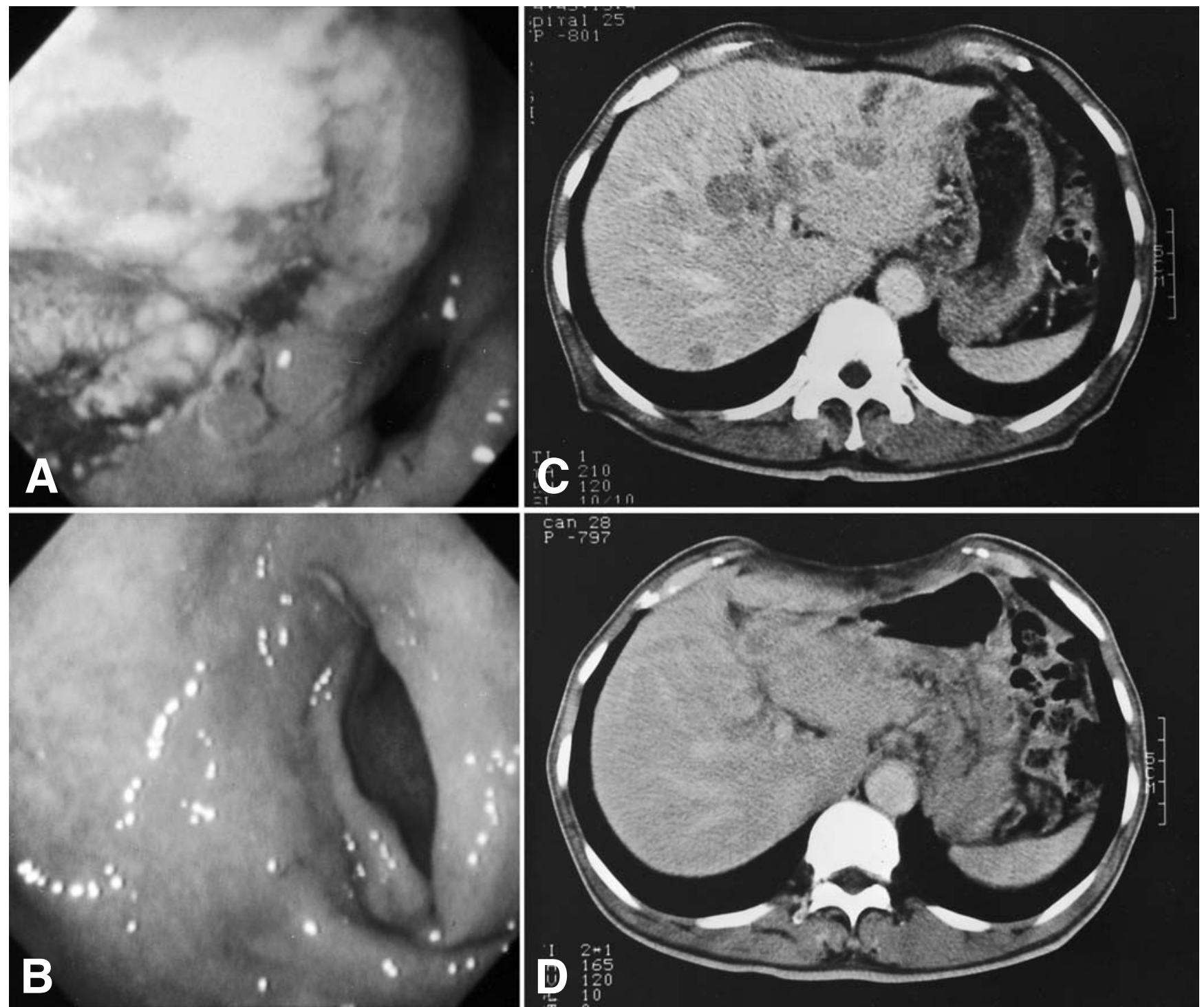

Fig. 1A,B. Gastrointestinal fiberscopy (GIF) demonstrated a type 2 advanced gastric carcinoma before treatment (A). After the third course of chemotherapy, only a gastric ulcer scar was observed (B). C,D Abdominal computed tomography (CT) showed multiple liver metastases in the bilateral lobes $(\mathbf{C})$. The lesions had regressed markedly after treatment $(\mathbf{D})$

\section{Case report 1}

A 69-year old man, who had complained of upper abdominal discomfort for a month, was referred to the Tottori University Hospital. On physical examination, a tumor was palpable in the epigastric region. Gastrointestinal fiberscopy (GIF) and upper gastrointestinal series (UGI) showed a type 2 advanced gastric carcinoma, with sharply demarcated and raised margins, on the anterior wall in the antrum (Fig. 1A). A biopsy specimen showed moderately differentiated tubular adenocarcinoma. CT and USG showed multiple liver metastatic lesions in the bilateral lobes, all of which were below $5 \mathrm{~cm}$ (Fig. 1C). These examinations also showed that the gastric tumor had invaded the regional lymph nodes. The patient was given a diagnosis of stage IV gastric cancer (cT3, cP0, cH1, cN2, cM0) according to the Japanese classification of gastric carcinoma, 2nd English edition [21]. The PS of this patient was grade 0, and laboratory examination results were within the normal ranges. Because curative resection was impossible for this patient, the combination chemotherapy of S-1 and low-dose CDDP was started.

S-1, $100 \mathrm{mg}$, was administered orally every day for 28 days, followed by 14 days' rest, as one course. As the biochemical modulator, $5 \mathrm{mg}$ of CDDP was infused three times each week (days $1,3,5$ ) during S-1 administration. One course resulted in a marked reduction of 
the tumor. GIF revealed a small ulcer without raised margins. On the abdominal CT scan after treatment, the liver metastases and regional lymph nodes had decreased in size (Fig. 1C). Only grade 1 nausea was observed during the chemotherapy. Therefore, we concluded that continuation of the combination chemotherapy at the outpatient clinic would be beneficial. After the third course, the effectiveness was evaluated by GIF, CT, and USG. Endoscopy revealed that the type 2 tumor had changed to an ulcer scar (Fig. 1B). Although the chemotherapy was markedly effective for this patient, small but multiple liver metastases were revealed by CT scan.

During the fourth course, 5 months after the first administration, liver metastases were confirmed as regrowing. Thus, we changed the regimen for this patient to the second-line chemotherapy.

\section{Case report 2}

A 67-year-old woman with a 2-month history of appetite loss and epigastric discomfort was referred to the
Tottori University Hospital. GIF and UGI demonstrated a type 4 advanced gastric carcinoma invading the duodenal bulb. A biopsy specimen showed poorly differentiated adenocarcinoma. An abdominal CT scan showed swelling of the regional and paraaortic lymph nodes and at least four metastatic lesions in the liver (Fig. 2A,B). The patient was diagnosed with advanced disease (cT3, cP0, cH1, cN3, cM0; stage IV).

$\mathrm{S}-1,120 \mathrm{mg}$, was administered orally every day for 28 days, followed by 14 days' rest, as one course. CDDP $10 \mathrm{mg}$ was infused three times each week (days $1,3,5$ ) during S-1 administration. Two courses resulted in the complete disappearance of the swelling in the paraaortic lymph nodes. The metastatic lesions of the liver and regional lymph nodes had decreased in size (Fig. 2C,D), but the primary tumor was evaluated as "no change" (NC). Because the liver metastases had shown marked reduction, we concluded that curative resection could be achieved, using microwave coagulation therapy for the liver metastases.

Three weeks after the completion of the chemotherapy, and 4 months after the first administration, the
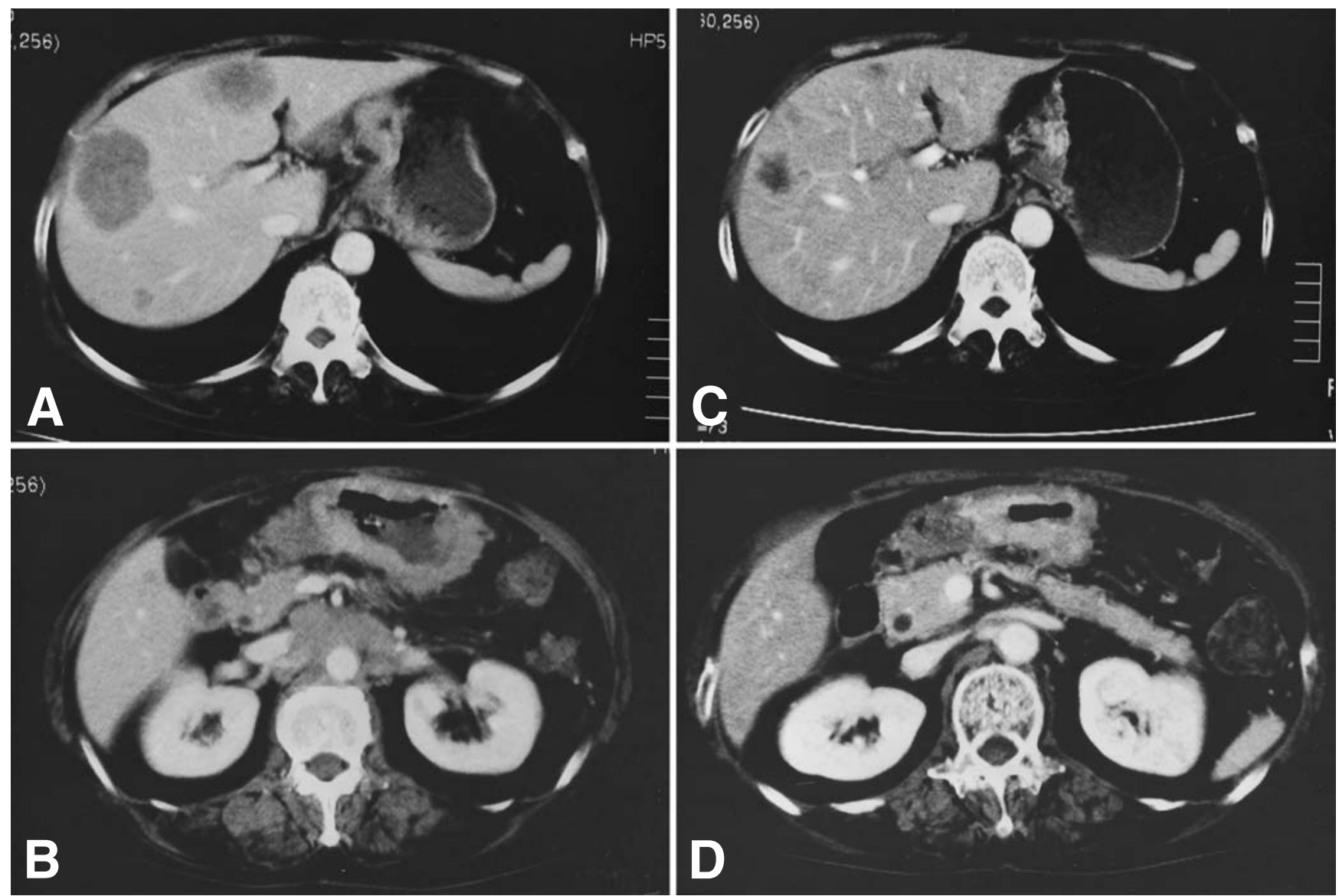

Fig. 2A-D. Abdominal CT scans demonstrated metastases in the liver (A) and paraaortic lymph nodes (B). After treatment, metastatic lesions in the liver had decreased in size $(\mathbf{C})$, as had the swollen lymph nodes $(\mathbf{D})$ 
patient was operated on. Although the primary tumor and regional lymph nodes were resectable en bloc, small multiple metastatic lesions less than $1 \mathrm{~cm}$ diameter were detected on the surface of the bilateral lobes of the liver. Therefore, only palliative total gastrectomy was performed. After the operation, an other regimen was employed for this patient to control the hepatic lesions.

\section{Discussion}

The prognosis of patients with unresected and recurrent gastric cancer is extremely poor. Although advances in surgical techniques have improved the prognosis of these patients, only a few survivors have been reported even after complete resection. In particular, there are few surgical benefits for those patients with metastases to other organs or distant lymph nodes. Thus, there is a great need for the development of chemotherapy that will improve the prognosis of these patients. Because response rates to the major drugs used alone have been reported to be less than $25 \%$ [4-6], most patients with advanced gastric cancer are given combination therapies. According to a JCOG study, FP (5-FU combined with CDDP) therapy had a better response than 5-FU alone [25]. Continuous 5-FU with low-dose CDDP is widely used in Japan because the response rate to this regimen is equal to that of the original FP protocol, but with less toxicity [26]. Although low-dose FP therapy is available, no regimen yields better survival than continuous injection of 5-FU alone, but most patients need long hospitalization for systemic chemotherapy.

Shirasaka et al. [17] proposed a suitable formulation for a 5-FU derivative FT-based drug called S-1. This novel oral anticancer drug improves the tumor-selective toxicity of 5-FU, by the actions of two modulators, CDHP and Oxo. The phase II trials in Japan showed a high antitumor effect, and low toxicity $[19,20,27]$. Because 5-FU is widely included in study protocols of combined treatments for gastric cancer, the S-1-based combined regimens have been considered to merit consideration as an alternative treatment. Recently, the use of S-1 and low-dose CDDP was reported for several patients [28-30], and a multicenter clinical study is in progress.

We have tested a combination therapy of S-1 and low-dose CDDP for patients with advanced gastric cancer. In our patients, treatment with this combination therapy induced a response rate of $53 \%(8 / 15)$, which is similar to those of the phase II clinical trials of S-1 alone ( $54 \%$ in the early trial and $49 \%$ in the late trial). For site efficacy, the response rate was $50 \%(5 / 10)$ for the primary lesions, and 50\% (3/6) for liver metastases, and these rates were better than those in the early and late phase II clinical trials $(28 \%$ and $39 \%$ for the primary lesions, and $31 \%$ and $25 \%$ for liver metastases, respectively). S-1 tended to be more effective for lymph node metastases than for the primary lesion, lung metastases, or liver metastases in the phase II study. Moreover, the response rate for cervical lymph nodes $(68 \%)$ was higher than that for abdominal lymph nodes (49\%) [31]. In the present study, the response rate for lymph node metastases was $39 \%(5 / 13)$ while the prior studies revealed higher response rates $(69 \%$ and $57 \%)$. This difference is considered to be due to the fact that all of the evaluable lymph node metastases in our study were abdominal ones. Thus, S-1 combined with low-dose CDDP shows promise as having a potent anticancer effect on advanced gastric cancer. Because the response rate of this combination chemotherapy in our study was not superior to the results of S-1 alone, the promising effect of low-dose CDDP for the biochemical modulation of 5-FU should be confirmed in a large randomized trial comparing S-1/low-dose CDDP and S-1 alone.

A phase I/II study of $\mathrm{S}-1$ plus high-dose CDDP showed a high response rate $(19 / 25 ; 76 \%)$. However, in that trial, adverse reactions were frequently observed [32]. In the current study, the incidence of adverse reactions was $60 \%(9 / 15)$. Although grade 3 toxicity was observed in 2 patients (13\%), grade 4 toxicity was not encountered. This rate seems to be comparable with that of the previous phase II trials $(20 \%)$. This result may indicate that there is now sufficient experience to control the adverse toxicity in S-1 treatment. Gastrointestinal adverse reactions appeared in $40 \%$ of patients in our study (6/15), and all of the reactions were grades 1 or 2 . These results indicate acceptable safety in the administration of S-1 combined with the infusion of CDDP.

In a setting of neoadjuvant chemotherapy, the regimen is required not only to induce a high response rate for improvement of resectability of the tumor but also to not induce severe toxicity that may delay surgery. It has been reported that some patients who received intensive neoadjuvant chemotherapy could not be operated on due to severe toxicity [33,34]. While $S-1$ is effective for recurrent gastric cancer, it is expected to be useful as neoadjuvant chemotherapy because of its mild toxicity. Yoshikawa et al. [35] reported a patient with highly invasive gastric cancer who had undergone complete resection after neoadjuvant chemotherapy with S-1. In that patient, although the tumor had invaded the duodenum, pancreas, and transverse colon, involving the regional lymph nodes, the primary tumor was markedly reduced due to the chemotherapy. Another case report of the use of S-1 as neoadjuvant chemotherapy noted that curative resection was successfully performed because of the complete disappearance of metastases in the Virchow's and paraaortic lymph nodes [31]. 
In our studies of $\mathrm{S}-1$ combined with low-dose CDDP as neoadjuvant chemotherapy, complete resection of advanced gastric cancer has not been performed. However, this regimen showed remarkable antitumor effects in some patients. Therefore, S-1 in combination with other drugs is expected to be a useful chemotherapy for advanced gastric cancer.

\section{References}

1. Kelsen DP. Cancer of the stomach. In: DeVita VT, editors. Cancer, principles and practice of oncology. 4th Ed. Philadelphia: JB Lippincott; 1995. pp 838-48.

2. Wils J. The treatment of advanced gastric cancer. Semin Oncol 1996;23:397-406.

3. Sakata Y, Suzuki H. Do we have a strategy for gastrointestinal cancers at present and the future? Cancer Chemother 1995;10: 169-80.

4. Moertel CG. Chemotherapy of gastrointestinal cancer. In: Tagnon HJ, Staquest MJ, editors. Recent advances in cancer treatment. New York: Raven; 1977. pp 311-23.

5. Beer M, Cocconi G, Ceci G, Varini M, Cavalli F. A phase II study of cisplatin in advanced gastric cancer. Eur J Cancer Clin Oncol 1983;19:717-20.

6. Miyamoto K, Yoshida S, Saito D, Shimada Y, Tajiri H, Yamaguchi $\mathrm{H}$, et al. Pilot phase II study of cisdiaminedichloroplatinum (II) against metastatic gastric cancers. Jpn J Clin Oncol 1990;20:169-76.

7. Klein HO, Dias Wickramanayake P, Dieterle F, Mohr R, Oerkermann $\mathrm{H}$, Brock J, et al. Chemotherapieprotokoll zur behandlung des metastasierenden Magenkarzinoms. Methitrexat, Adriamycin, und 5-fluorouracil. Dtsch Med Wochenschr 1982; 107:1708-12.

8. Webb A, Cunningham D, Scarffe JH, Harper P, Norman A, Joffe $\mathrm{JK}$, et al. Randomized trial comparing epirubicin, cisplatin, and fluorouracil versus fluorouracil, doxorubin, and methotrexate in esophagogastric cancer. J Clin Oncol 1997;15:261-7.

9. Wils JA, Klein HO, Wagerer DJT, Bleiberg H, Reis H, Korsten F et al. Sequential high-dose methotrexate and fluorouracil combined with doxorubicin: a step ahead in the treatment of gastric cancer: a trial of the EORTG. J Clin Oncol 1991;9:827-31.

10. Kelsen D, Atiq OT, Saltz L, Niedzwiecki D, Ginn D, Chapman D, et al. FAMTX versus etoposide, doxorubicin, and cisplatin: a random assignment trial in gastric cancer. J Clin Oncol 1992;10: $541-8$.

11. Preusser P, Wilke H, Achterrath W, Fink U, Lenaz L, Heinicke A, et al. Phase II study with the combination of etoposide, doxorubicin, and cisplatin in advanced measurable gastric cancer. $\mathrm{J}$ Clin Oncol 1989;7:1310-17.

12. Lerner A, Gonin R, Steele GD Jr, Mayer J. Etoposide, doxorubicin, and cisplatin chemotherapy for advanced gastric adenocarcinoma: results of a phase II trial. J Clin Oncol 1992;10:53640.

13. Lacave AJ, Baron FJ, Anton LM, Estrada E, De Sande LM, Palacio I, et al. Combination chemotherapy with cisplatin and 5-fluorouracil 5-day infusion in the therapy of advanced gastric cancer: a phase II trial. Ann Oncol 1991;2:751-4.

14. Rougier P, Ducreux M, Mahjoubi M, Pignon JP, Bellefqih S, Oliveira J, et al. Efficacy of combined 5-fluorouracil and cisplatinum in advanced gastric carcinomas: a phase II trial with prognostic factor analysis. Eur J Cancer 1994;30:12639.

15. Vanhoefer U, Rougier P, Wilke H, Ducreux MP, Lacave AJ, Cutsem EV, et al. Final results of randomized phase III trial of sequential high-dose methotrexate, fluorouracil, and doxorubicin versus etoposide, leucovorin, and fluorouracil versus infusional fluorouracil and cisplatin in advanced gastric cancer: a trial of the EORTG. J Clin Oncol 2000;18:2648-57.

16. Shirasaka T, Nakano K, Takechi T, Satake H, Uchida J, Fujioka A, et al. Antitumor activity of $1 \mathrm{M}$ tegafur - $0.4 \mathrm{M}$ 5-chloro-2,4dihydroxypyridine $-1 \mathrm{M}$ potassium oxonate (S-1) against human colon carcinoma orthotopically implanted into nude rats. Cancer Res 1996;56:2602-6.

17. Shirasaka T, Shimamoto S, Ohshimo H, Yamaguchi M, Kato T, Yonekura K, et al. Development of a novel form of an oral 5fluorouracil derivative (S-1) directed to the potentiation of the tumor selective cytotoxicity of 5-fluorouracil by two biochemical modulators. Anticancer Drugs 1996;7:548-57.

18. Takechi T, Nakano K, Uchida J, Mita A, Toko K, Takeda S, et al. Antitumor activity and low intestinal toxicity of S-1, a new formulation of oral tegafur, in experimental tumor models in rats. Cancer Chemother Pharmacol 1997;39:205-11.

19. Sugimachi K, Maehara Y, Horikoshi N, Shimada Y, Sakata Y, Mitachi Y, et al. An early phase II study of oral S-1, a newly developed 5-fluorouracil derivative for advanced and recurrent gastrointestinal cancers. Oncology 1999;57:202-10.

20. Sakata Y, Ohtsu A, Horikoshi N, Sugimachi K, Mitachi Y, Taguchi T. Late phase II study of novel oral fluoropyrimidine anticancer drug S-1 (1 M tegafur $-0.4 \mathrm{M}$ otastat potassium) in advanced gastric cancer patients. Eur J Cancer 1998;34:171520.

21. Japanese Gastric Cancer Association. Japanese classification of gastric carcinoma, 2nd English edition. Gastric Cancer 1998;1:1024.

22. World Health Organization. WHO handbook for reporting results of cancer treatment. WHO offset publication no. 48. Geneva: World Health Organization; 1979.

23. Japanese Research Society for Gastric Cancer, editors. Japanese classification of gastric carcinoma. 1st English Ed. Tokyo: Kanehara; 1995.

24. Tobinai K, Kohno A, Shimada Y, Watanabe T, Tamura T, Takeyama K, et al. Toxicity grading criteria of Japan Clinical Oncology Group. Jpn J Clin Oncol 1993;23:250-7.

25. Shimada Y, Shirao K, Ohtsu A, Hyodo I, Saito H, Yamanishi N, et al. Phase III study of UFT + MMC versus 5-FU + CDDP versus 5-FU alone in patients with advanced gastric cancer. Proc Am Soc Clin Oncol 1999;18:1043.

26. Chung YS, Yamashita Y, Nakata B, Nitta A, Inoue T, Hirayama $\mathrm{K}$, et al. Combination therapy of 5-FU and low dose CDDP for advanced and recurrent gastric cancer. Jpn J Cancer Chemother 1995;22:149-51.

27. Koizumi W, Kurihara M, Nakano S, Hasegawa K. Phase II study of S-1, a novel oral derivative of 5-fluorouracil, in advanced gastric cancer. Oncology 2000;58:191-7.

28. Saikawa Y, Kanai T, Kawano Y, Otani Y, Kubota T, Kitajima M. A novel combined chemotherapy using TS-1 and low-dose cisplatin against liver metastasis of gastric cancer. Jpn J Cancer Chemother 2002;29:1241-5.

29. Gomi T, Ohtoshi M, Tamaki N, Momoi H, Kanaya S, Katayama $\mathrm{T}$, et al. Three cases of advanced gastric cancer treated by TS- 1 in combination with low-dose cisplatin. Jpn J Cancer Chemother 2002;29:301-4.

30. Kamata T, Hayashi Y, Minatoya G, Michiwa Y, Onishi I, Takeda $\mathrm{T}$, et al. A pilot study of low-dose TS-1 and cisplatin combination chemotherapy for advanced gastric cancer. Jpn J Cancer Chemother 2001;28:1419-22.

31. Iwazawa T, Kinuta M, Yano H, Matsui S, Tamagaki S, Yasue A, et al. An oral anticancer drug, TS-1, enabled a patient with advanced gastric cancer with Virchow's metastasis to receive curative resection. Gastric Cancer 2002;5:96-101.

32. Ohtsu A, Boku N, Nagashima F, Koizumi W, Tanabe S, Saigenji $\mathrm{K}$, et al. A phase I/II study of S-1 plus cisplatin in patients with advanced gastric cancer (abstract). Proc Am Soc Clin Oncol 2001;20:165. 
33. Yonemura Y, Sawa T, Kinoshita K, Matsuki N, Fushida S, Tanaka S, et al. Neoadjuvant chemotherapy for high-grade advanced gastric cancer. World J Surg 1993;17:256-61.

34. Nakajima T, Ota K, Ishihara S, Oyama S, Nishi M, Ohashi Y, et al. Combined intensive chemotherapy and radical surgery for incurable gastric cancer. Ann Surg Oncol 1997;4:203-8.
35. Yoshikawa T, Kanari M, Tsuburaya A, Kobayashi O, Sairenji M, Motohashi $\mathrm{H}$, et al. Advanced gastric carcinoma successfully treated with TS-1 as neoadjuvant chemotherapy. Gastric Cancer 2000;3:171-5. 